\title{
Evolution of Initial Pharmacologic Treatment of Newly Diagnosed Parkinson's Disease Patients over a Decade in Singapore
}

\author{
Shermyn Neo $\mathbb{D}^{1},{ }^{1}$ Sheng Yong Aidan Wong, ${ }^{2}$ Hwee Lan $\mathrm{Ng},{ }^{1}$ Wei Li, ${ }^{1}$ Kay Yaw Tay, ${ }^{1,3}$ \\ Wing Lok Au, ${ }^{1,3}$ and Louis Chew Seng Tan $\left(^{1,3}\right.$ \\ ${ }^{1}$ Department of Neurology, National Neuroscience Institute, Singapore \\ ${ }^{2}$ Department of Research, National Neuroscience Institute, Singapore \\ ${ }^{3}$ Duke-NUS Medical School, Singapore \\ Correspondence should be addressed to Shermyn Neo; shermyn.neo.x.m@singhealth.com.sg
}

Received 8 January 2020; Accepted 11 March 2020; Published 30 March 2020

Academic Editor: Carlo Ferrarese

Copyright (C) 2020 Shermyn Neo et al. This is an open access article distributed under the Creative Commons Attribution License, which permits unrestricted use, distribution, and reproduction in any medium, provided the original work is properly cited.

Objective. The aim of this study is to compare Parkinson's disease (PD) treatment practices by movement disorder (MD) specialists across a decade, and to determine the factors that influence drug choice for the motor symptoms of PD in newly diagnosed drug-naïve patients. Methods. This prospective temporal analysis included patients seen at the National Neuroscience Institute in Singapore and diagnosed with PD by MD specialists in the years 2007 and 2017. Primary outcomes were use of specific PD drugs and changes in drug-prescribing patterns. Descriptive analyses and multivariable logistic regression models determined the extent to which patient characteristics were associated with type of PD treatment. Results. Of 230 patients with PD (mean (SD) age, 66.7 (10.3) years), 131 (57.0\%) were male. From 2007 to 2017, the use of ergot dopamine agonists and anticholinergics decreased from $19.3 \%$ to $2.0 \%(P<0.001)$ and from $12.0 \%$ to $2.7 \%(P=0.004)$, respectively. The use of monoamine oxidase $\mathrm{B}$ inhibitors $(\mathrm{MAOBI})$ increased from $13.3 \%$ to $25.2 \%(P=0.033)$. The use of levodopa (LD)-sparing strategies decreased nonsignificantly from $33.7 \%$ to $24.5 \%(P=0.133)$. Overall, $196(85.2 \%)$ patients were initiated on symptomatic monotherapy, with LD being the most commonly prescribed. MAOBI was the most common drug used in combination therapy. Age $\leq 70$ (adjusted OR, 11.9; 95\% CI, 4.5-31.5) and Hoehn and Yahr (HY) stage <2 (adjusted OR, 3.4; 95\% CI, 1.5-7.7) were independent factors for LDsparing strategies. Non-LD prescriptions (13 of 92; 14.1\%) were more likely to be discontinued compared to LD ones (6 of 149; 4.0\%) $(P=0.005)$. Conclusions. Drug-prescribing patterns in PD have changed significantly through the last decade, influenced by emerging evidence and reports of adverse drug effects. Choosing drugs based on the patient's age and disease severity remain sound guiding principles across the years. It is important that international and national guidelines for pharmacotherapy in PD be updated consistently throughout different socioeconomic settings to optimize care.

\section{Introduction}

Treatment of Parkinson's disease (PD), the second most common neurodegenerative disorder worldwide [1], is largely symptomatic, with its most effective oral therapeutic agent still being levodopa (LD), despite being more than half a century old. The optimal choice of initial symptomatic treatment, however, is unclear, with scientific literature and pivotal clinical trials swinging expert opinion at various turns and igniting fierce debate between $\mathrm{LD}$ proponents and those who prefer a LD-sparing strategy $[2,3]$. Drug-prescribing patterns may be guided by these evolving scientific evidences.

Few data on temporal trends in Parkinson drugprescribing patterns in drug-naïve PD patients exist. Most available studies on drug-prescribing patterns in PD have been cross-sectional analyses or use the drug tracer methodology, making it difficult to identify changing patterns in PD drug utilization or patient factors that influence the choice of drug [4-6]. In studies with longitudinal data, 
analyses were on patients with a varied duration of illness; thus, were not designed to answer which dopaminergic replacement strategy was preferred by physicians as initial therapy $[7,8]$.

The aim of this study is to compare PD treatment practices by movement disorder (MD) specialists across a decade and to determine the factors that influence drug choice in newly diagnosed drug-naïve PD patients in Singapore. A 10-year comparison was felt to be appropriate as many important pragmatic real-world studies had emerged in the intervening decade $[9,10]$ with potential impact on drug-prescribing practices.

\section{Materials and Methods}

This study was approved by the Centralized Institution Review Board of the Singapore Health Services.

2.1. Data Source. A Movement Disorders database with prospectively collected information has been in existence since 2002 at the National Neuroscience Institute in Singapore (NNI). Patients seen at NNI and diagnosed by MD specialists according to the National Institute of Neurological Disorders and Stroke (NINDS) diagnostic criteria for PD [11] in the years 2007 and 2017 were evaluated. The year 2007 was selected as the dopamine agonists, ropinirole, and pramipexole were only available in the local hospitals 2 years prior. Patients had to be (i) seen by a MD specialist within 1 year of diagnosis, (ii) drug-naïve, and (iii) started on medications within the first 2 clinic visits.

2.2. Data Collection. Demographic and clinical information such as age, sex, date of diagnosis, modified Hoehn and Yahr (HY) stage and presence of rest tremor prior to commencement of PD drug, and initial PD drug prescriptions were recorded. For the purpose of this study, any discontinuation of medication(s) within the timeframe of 2 subsequent follow-up clinic visits that occurred within 2 years of the first visit was assumed to be due to medication intolerance. While reasons for discontinuation of treatment were not available for individual patients, it was presumed that medication sideeffects usually occur within the first few weeks to months of initiating therapy and is a common reason for discontinuation of therapy (compared to inadequate therapy, in case of which physicians would usually increase the dosage of medication or add on another medication). To ensure that medications were not discontinued because patients defaulted on follow-up visits, their subsequent 2 clinic visits had to occur within 2 years of the index visit. Patients diagnosed in 2017 who had only 1 follow-up visit at the point of study data collection were excluded from this portion of analysis.

From 2007 to 2017, the available oral DA at our institution was bromocriptine, as well as immediate release and sustained release forms of both ropinirole and pramipexole. Available LD formulations included Madopar, Madopar
HBS, Sinemet, Sinemet CR, and Credanil. Up to 2017, the only available monoamine oxidase B inhibitor (MAOBI) in the country was selegiline. Total levodopa equivalent dose (LED) per day was calculated for each patient, using standardized conversion formulae.

2.3. Statistical Analysis. Statistical analysis was performed using SPSS v21. Descriptive statistics summarized all variables overall, by the year of diagnosis and by the treatment type. One-way ANOVA was used to assess associations between continuous variables of interest and use of a LDsparing strategy, while Chi-square and Fisher's exact tests were used to assess similar associations with categorical variables. A multiple logistic regression model, with age, sex, HY stage, duration of illness, and presence of rest tremor as covariates, was used to determine independent variables favouring LD-sparing strategies. Linear regression analysis, using the same covariates, was performed to assess factors affecting total daily LED. All statistical tests assumed a 2sided type one error rate of 0.05 .

\section{Results}

3.1. Baseline Characteristics. A total of 230 patients met eligibility criteria and were included in the study. Table 1 summarizes the baseline demographic and clinical characteristics of the study population, cohorted by the year of diagnosis. The mean (SD) age of our patients was 66.7 (10.3) years, and 131 (57.0\%) were male. The mean (SD) HY stage prior to commencement of PD drug was 2.3 (0.7). Patients' age, sex, HY stage, duration of illness, and presence of rest tremor at baseline did not differ in the 2007 and 2017 cohorts.

3.2. Treatment Patterns in 2007 and 2017. As shown in Table 1 and Figure 1, the use of ergot DA and anticholinergics decreased from $19.3 \%$ to $2.0 \%(P<0.001)$ and from $12.0 \%$ to $2.7 \%(P=0.004)$, respectively, in 2017 , compared to 2007. The use of MAOBI, on the other hand, increased from $13.3 \%$ in 2007 to $25.2 \%$ in $2017(P=0.033)$. The use of LD-sparing strategies decreased nonsignificantly from $33.7 \%$ in 2007 to $24.5 \%$ in $2017(P=0.133)$. The mean daily LED was similar in 2007 and 2017 (198 mg/day vs. $207 \mathrm{mg} /$ day; $P=0.795)$.

3.3. Drug Combinations. Across both 2007 and 2017 cohorts, 196 (85.2\%) patients were initiated on symptomatic monotherapy, with LD being the most commonly prescribed monotherapy $(n=151 ; 77.0 \%)$. Table 2 shows the different drug combinations of patients on dual or triple therapy. MAOBI were most likely PD drugs to be used in combination therapies, with 26 of 34 patients on them.

3.4. Factors Affecting Use of LD vs. LD-Sparing Strategies. Compared to LD users, patients on LD-sparing strategies were younger $(P<0.001)$ and had milder disease $(P<0.001)$ (see Table 3). In a multiple logistic regression model, age $\leq 70$ 
TABLE 1: Patient characteristics and treatment based on the year of diagnosis.

\begin{tabular}{|c|c|c|c|c|}
\hline & Entire cohort $n=230$ & 2007 cohort $n=83$ & 2017 cohort $n=147$ & $P$ value \\
\hline Mean (SD) age, years & $66.7(10.3)$ & $66.4(10.1)$ & $66.9(10.5)$ & 0.737 \\
\hline Male, $n(\%)$ & $131(57.0)$ & $48(57.8)$ & $83(56.5)$ & 0.840 \\
\hline Mean (SD) HY stage & $2.3(0.7) ; n=222$ & $2.4(0.7) ; n=76$ & $2.2(0.6) ; n=146$ & 0.196 \\
\hline Mean (SD) duration of illness, months & $65.5(57.0) ; n=228$ & $59.7(56.4) ; n=82$ & $68.6(57.3) ; n=146$ & 0.246 \\
\hline Rest tremor present & $125(61.3) ; n=204$ & $44(69.8) ; n=63$ & $81(57.4) ; n=141$ & 0.093 \\
\hline Monotherapy, $n(\%)$ & $196(85.2)$ & $70(84.3)$ & $126(85.7)$ & 0.778 \\
\hline \multicolumn{5}{|l|}{ Treatment, $n(\%)$} \\
\hline \multicolumn{5}{|l|}{ LD } \\
\hline $\begin{array}{l}\text { Total* } \\
\text { Monotherapy }\end{array}$ & $\begin{array}{l}166(72.2) \\
151(65.7) \\
\end{array}$ & $\begin{array}{l}55(66.3) \\
53(63.9) \\
\end{array}$ & $\begin{array}{c}111(75.5) \\
98(66.7)\end{array}$ & 0.133 \\
\hline $\begin{array}{l}\text { Ergot DA } \\
\text { Total } \\
\text { Monotherapy }\end{array}$ & $\begin{array}{l}19(8.3) \\
10(4.3)\end{array}$ & $\begin{array}{l}16(19.3) \\
10(12.0)\end{array}$ & $\begin{array}{l}3(2.0) \\
0(0.0)\end{array}$ & $<.001$ \\
\hline $\begin{array}{l}\text { Nonergot DA } \\
\text { Total }^{*} \\
\text { Monotherapy }\end{array}$ & $\begin{array}{l}18(7.8) \\
10(4.3)\end{array}$ & $\begin{array}{l}4(4.8) \\
1(1.2)\end{array}$ & $\begin{array}{c}14(9.5) \\
9(6.1)\end{array}$ & 0.202 \\
\hline $\begin{array}{l}\text { MAOBI } \\
\text { Total }^{*} \\
\text { Monotherapy }\end{array}$ & $\begin{array}{c}48(20.9) \\
22(9.6)\end{array}$ & $\begin{aligned} & 11(13.3) \\
& 4(4.8) \\
&\end{aligned}$ & $\begin{array}{l}37(25.2) \\
18(12.2) \\
\end{array}$ & 0.033 \\
\hline $\begin{array}{l}\text { Anticholinergic } \\
\text { Total* } \\
\text { Monotherapy }\end{array}$ & $\begin{array}{c}14(6.1) \\
3(1.3) \\
\end{array}$ & $\begin{aligned} & 10(12.0) \\
& 2(2.4) \\
&\end{aligned}$ & $\begin{array}{l}4(2.7) \\
1(.07) \\
\end{array}$ & 0.004 \\
\hline Mean (SD) LED, mg/day & $204(113)$ & $198(106)$ & $207(117)$ & 0.566 \\
\hline
\end{tabular}

DA, dopamine agonist; HY, Hoehn and Yahr; LD, levodopa; LED, levodopa equivalent dose; MAOBI, monoamine oxidase type B inhibitor; SD, standard deviation. ${ }^{*}$ Combination and monotherapy.

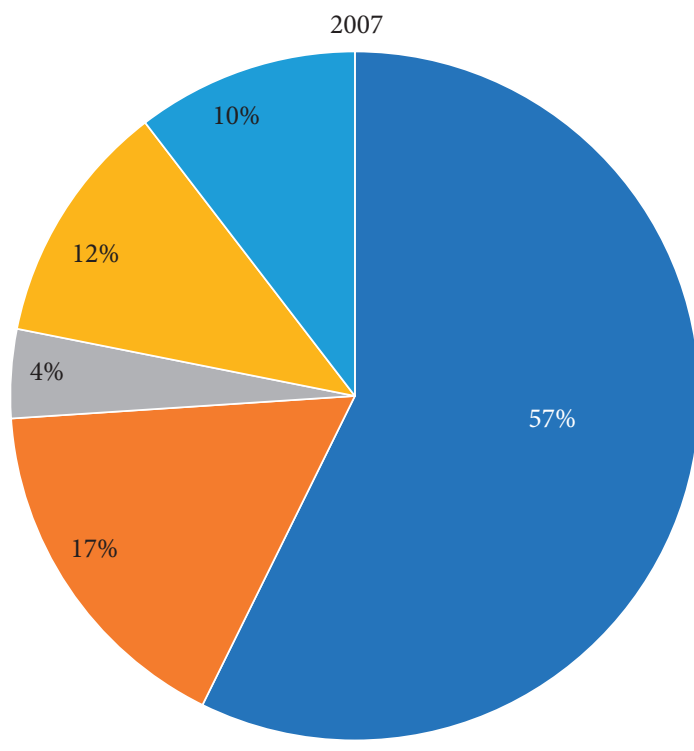

LD

Ergot DA

- Nonergot DA

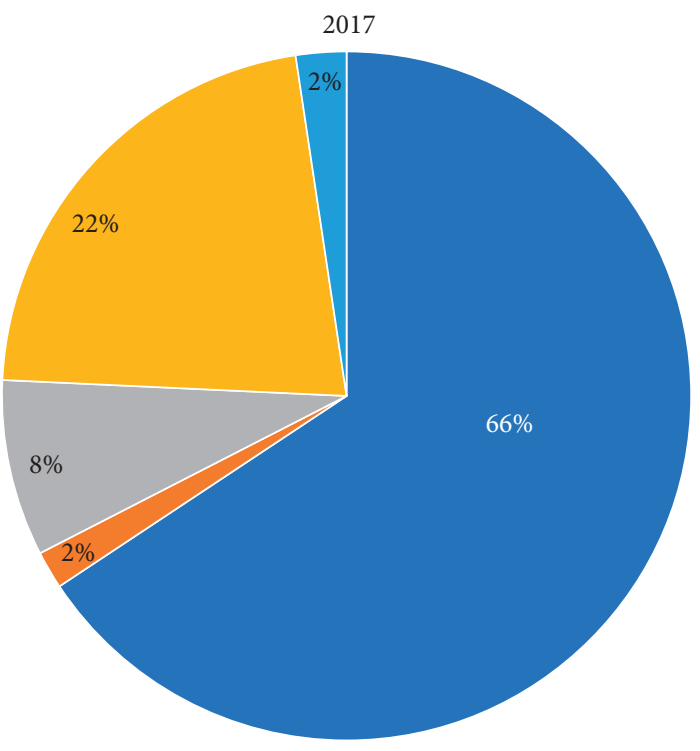

MAOBI

Anticholinergic

Figure 1: PD drug treatment by year.

(adjusted OR, 11.9; 95\% CI, 4.5-31.5) and HY stage <2 (adjusted OR, 3.4; 95\% CI, 1.5-7.7) were independent factors for LD-sparing strategies, after adjusting for sex, duration of illness, and presence of rest tremor. The associations remained significant, after sensitivity analysis by the year of diagnosis. 
TABle 2: Drug combinations for patients on dual/triple therapy in 2007 and 2017.

\begin{tabular}{lc}
\hline Drug combinations & $n=34$ \\
\hline Dual therapy & \\
LD + DA & 2 \\
LD + MAOBI & 10 \\
LD + anticholinergic & 2 \\
DA + MAOBI & 10 \\
DA + anticholinergic & 4 \\
MAOBI + anticholinergic & 5 \\
\hline Triple therapy & \\
LD + DA + MAOI & 1 \\
\hline
\end{tabular}

DA, dopamine agonist; LD, levodopa; MAOBI, monoamine oxidase type B inhibitor.

TABLE 3: Univariate analysis of factors determining the use of LD vs. LD-sparing strategies.

\begin{tabular}{lccc}
\hline & LD & $\begin{array}{c}\text { LD- } \\
\text { sparing }\end{array}$ & $\begin{array}{c}P \\
\text { value }\end{array}$ \\
\hline Age & $70.3 \pm 8.8$ & $57.5 \pm 8.1$ & $<.001$ \\
Male, $n$ (\%) & $91(54.8)$ & $40(62.5)$ & 0.292 \\
HY stage, mean (SD) & $2.4(0.7)$ & $1.9(0.5)$ & $<.001$ \\
Mean duration of illness (SD), & $68.9(61.2)$ & $56.8(43.6)$ & 0.148 \\
months & $84(57.5)$ & $41(70.7)$ & 0.082 \\
Rest tremor present, $n(\%)$ &
\end{tabular}

HY, Hoehn and Yahr; LD, levodopa. "All characteristics were analyzed as continuous variables except sex and presence of rest tremor. Results of multivariate analysis including all factors listed in the table are reported in the main text.

3.5. Factors Affecting Total Daily LED. Table 4 shows the effect of age, sex, HY stage, duration of illness, and presence of rest tremor on LED in the combined cohort. Linear regression analysis demonstrated that every increase of $1 \mathrm{HY}$ stage was associated with a corresponding increase of 52 units in $\operatorname{LED}(P<0.001)$.

3.6. Discontinuation of Treatment. Non-LD prescriptions (13 of $92 ; 14.1 \%$ ) were more likely to be discontinued compared to LD ones (6 of $149 ; 4.0 \%)(P=0.005)$. Eight of the discontinued therapies in the non-LD group were MAOBI, while DA accounted for the remaining 5.

\section{Discussion}

In this prospective temporal analysis of drug-naïve PD patients who initiated PD medication, it was observed that the use of ergot DA and anticholinergics had declined in the decade from 2007 to 2017 . This was accompanied by a rise in the use of MAOBI, specifically selegiline.

The ergot DA have largely fallen out of use in view of reports of rare but devastating complications of fibrosis, in particular of cardiac valves. While cabergoline and pergolide are associated with the highest risks of fibrotic cardiac valve reactions [12], the same warning to limit dosage of DA and monitor patients for signs of fibrosis while on treatment has been extended to all ergot-derived DA by the European Medicines Agency in 2008.
TABLE 4: Factors affecting total daily LED.

\begin{tabular}{lcc}
\hline & Effect estimate & $P$ value \\
\hline Age & 1.312 & 0.085 \\
Male & 9.612 & 0.533 \\
HY stage & 51.626 & $<.001$ \\
Duration of illness & -3.777 & 0.806 \\
Presence of rest tremor & -0.67 & 0.636 \\
\hline
\end{tabular}

HY, Hoehn and Yahr.

Anticholinergics with their poor side-effect profile has never been a drug of preference in PD patients, who tend to be older. Greater awareness that anticholinergic use in PD patients is associated with increased risk of adverse health outcomes including fractures, delirium, and hospitalization [13] is likely to have contributed to its decreased usage. In addition, it is increasingly recognized that $\mathrm{PD}$ patients may have cognitive impairment even early in their disease course [14]. A recent study showed that inappropriate prescribing patterns are common in older PD patients who may be on anticholinergics and dementia treatment at the same time [15].

MAOBI use in PD rose to prominence at the turn of the millennium, with selegiline postulated to have a possible neuroprotective effect based on its capacity to inhibit MAO-B oxidation of MPTP to MPP + which is toxic to dopaminergic neurons [16]. Furthermore, by blocking free radical formation from the oxidation of dopamine, it is believed that selegiline can slow down neuronal degeneration in PD [17]. Finally, its metabolite, desmethylselegiline, is thought to have antiapoptotic properties [18]. Evidence of its neuroprotective effects in in vitro and in vivo laboratory models led to expert panels recommending the use of selegiline in early PD [19]. Unfortunately, the DATATOP [20] and SINDEPAR [21] trials undertaken to demonstrate this disease-modifying effect have been hampered by confounding symptomatic effects of the antiparkinsonian medications which can have long duration responses that persist for weeks after withdrawal. Nonetheless, the prevailing expert opinion might have led to increasing selegiline prescriptions at our center. This is supported by the finding that the greatest increase in usage of MAOBI was in patients who had also initiated LD. As LD has a far superior symptomatic effect, it may be postulated that the MAOBI was prescribed by our MD specialists for its putative neuroprotective effect. Our center's positive experience with selegiline is reflected in a previous study published in 2011 which found that patients with early PD treated with selegiline for 3 years or more had slower progression of disease as evaluated by HY transition times [22]. Increased utilization of MAOBI was also observed in a recent study across 23 expert care centers [7], with the authors citing good safety profile and ease of use as possible reasons. Rasagiline at a dose of $1 \mathrm{mg}$ per day was showed in an elegantly designed delayed-start trial to have possible disease-modifying effect, but treatment with the $2 \mathrm{mg}$ dose failed to replicate the same result [23]; thus, the U.S. Food and Drug Administration did not label it as such. Since the end of 2017, rasagiline has also been made available at our center. With a convenient once a day dosing, it is anticipated that MAOBI use in Singapore may continue to rise. 
Interestingly, the increase in nonergot DA use at our center was not significant. There may be several reasons for this, with results of landmark studies being one. Both pramipexole and ropinirole-treated PD patients developed fewer motor complications then LD-treated ones [24, 25]. At the same time, there were increasing concerns of LD toxicity to the human substantia nigra via the mechanism of autooxidation and the generation of free radicals [26]. It was in this climate that national guidelines started to favor DA as initial symptomatic treatment $[19,27]$. The growing enthusiasm for DA was, however, dampened by reports of adverse drug effects in the ensuing decade. A case series documented 8 cases of PD patients on pramipexole or ropinirole falling asleep while driving [28]. This was followed by reports of ICD, which were confirmed by a large crosssectional study of $3090 \mathrm{PD}$ patients demonstrating that DA treatment was associated with a 2-3.5-fold increase in odds of having ICD [10]. Finally, PD-MED was an important pragmatic trial which showed that the long-term risk of developing dyskinesias in LD and LD-sparing groups were similar (36\% vs 33\%) with no difference in the occurrence of motor fluctuations between groups [9]. LD-treated patients in PD-MED enjoyed persistent benefits in mobility. A case for less emphasis on LD-induced dyskinesia has also been made by Chaudhuri et al. who noted that the prevalence of troublesome dyskinesia requiring treatment is low, with the use of deep brain stimulation and continuous drug-delivery strategies by transdermal or dopaminergic infusion therapies serving to reduce this further [29]. Thus, the subsequent negative literature (and conversely positive literature on LD) may account for the nonsignificant increase in nonergot DA use at our center.

In addition, external factors like cost could have also driven physician prescribing practices. LD remains the cheapest and most heavily government-subsidised PD drug in Singapore. Although ropinirole was subsequently subsided up to $50 \%$ in the early 2010 after losing its patent, it remains significantly more expensive than $\mathrm{LD}$, costing 10 times the price. Pramipexole is not subsidised in Singapore. Conversely, while selegiline is also not subsidised, it is considerably cheaper than the nonergot DA and approximates the cost of $\mathrm{LD}$, hence likely facilitating the rise in its usage. The continued use of the ergot DA, bromocriptine, despite its negative side-effect profile may also be explained by its low subsided cost. Although our study was not designed to study external factors influencing prescribing patterns, it is clear that scientific evidence alone does not drive behaviour in clinical practice. In a survey of neurologists on their perceived factors influencing choice of PD medication in Singapore, Tan et al. identified cost as an overriding factor in usage patterns [30]. The aforementioned study by Dubaz et al. also demonstrated no change in DA use between 2010 and 2017, although they did not make a distinction between ergot and nonergot DAs [7].

Daily LED did not differ in 2007 and 2017. This was not surprising as patients in both cohorts were similar in demographics and clinical profile. LED was influenced strongly by disease stage. Interestingly, sex did not affect LED, and both male and female patients received similar mean LED
$(192 \pm 117 \mathrm{mg} /$ day vs. $219 \pm 105 \mathrm{mg} /$ day; $P=0.064)$. Sex differences in treatment in PD are worth exploring, as several studies have reported that women are more likely to develop dyskinesias, with a lower body weight proposed as the cause [31]. If women and men are receiving similar doses, it may account for higher rates of this motor complication in women.

In our study, age and disease severity were found to be independent clinical factors that influenced whether a patient was initiated on LD or LD-sparing strategies, with older and more severely affected patients more likely to be on LD. This was consistent throughout the decade, supporting the observation that certain basic principles guided PD management by MD specialist at our center. While the debate on LD vs. LD-sparing strategies may not yet be resolved, considering these patient factors remain sound treatment principles. The superior side-effect profile and fewer concerns on LD-related motor complications [32] make LD the preferred choice in the elderly. Like previous studies, we demonstrated that non-LD treatment was not as well-tolerated as LD. In PD-MED, 28\% of DA-treated patients and $23 \%$ of MAOBI-treated patients discontinued allocated treatment due to side-effects, compared with $2 \%$ of LDtreated patients [9].

Our study has several limitations. As this is a single expert care center study in Singapore, the trends observed may not be generalizable to other settings. Our study did not collect nonmotor clinical factors, such as the presence of cognitive impairment or orthostatic hypotension, that are likely to influence to the physician's drug choice. This is because nonmotor symptoms (NMS) in PD were not widely appreciated until recently and documentation of NMS at baseline, prior to drug commencement, was not consistent in 2007. We were not able to study external factors like cost and government policy that may have important impact on PD drug-prescribing patterns. Finally, reasons for discontinuation of drug therapy were not available for individual patients, although care was taken to ensure that this was not due to patients being lost to follow-up. Nonetheless, this is the first study on temporal changes in prescribing patterns in drug-naïve PD patients and does shed light on how scientific evidence influences clinical practice. Our use of a current and comprehensive dataset that is updated regularly with clinical visit data is another strength.

In conclusion, drug-prescribing patterns in $\mathrm{PD}$ have changed significantly through the last decade with a shift away from LD-sparing strategies, possibly influenced by emerging evidence and postmarketing surveillance reports. Key guiding principles of choosing drugs based on the patient's age and disease severity remained consistent across the years. It is important that international and national guidelines for drug-prescribing in $\mathrm{PD}$ be updated and consistent throughout different socioeconomic settings to optimize care.

\section{Data Availability}

Data will be shared on request from any qualified investigator. 


\section{Conflicts of Interest}

The authors have no conflicts of interest.

\section{Acknowledgments}

This study was supported by the Singapore Ministry of Health's National Medical Research Council under its Translational and Clinical Research Flagship Programme (NMRC/TCR/013-NNI/2014) and Open Fund Large Collaborative Grant (MOH-OFLCG18May-0002).

\section{References}

[1] E. R. Dorsey and B. R. Bloem, "The Parkinson pandemic-a call to action," JAMA Neurology, vol. 75, no. 1, p. 9, 2018.

[2] A. J. Espay and A. E. Lang, "Common myths in the use of levodopa in Parkinson disease," JAMA Neurology, vol. 74, no. 6, p. 633, 2017.

[3] S.-Y. Lim, W. Poewe, and A. H. Tan, "Levodopa and Parkinson disease-myths revisited," JAMA Neurology, vol. 74, no. 10, p. 1270, 2017.

[4] M. M. Rosa, J. J. Ferreira, M. Coelho, R. Freire, and C. Sampaio, "Prescribing patterns of antiparkinsonian agents in Europe," Movement Disorders, vol. 25, no. 8, pp. 1053-1060, 2010.

[5] P. Surathi, N. Kamble, K. S. Bhalsing, R. Yadav, and P. K. Pal, "Prescribing pattern for Parkinson's disease in Indian community before referral to tertiary center," Canadian Journal of Neurological Sciences, vol. 44, no. 6, pp. 705-710, 2017.

[6] G. Trifirò, R. Savica, L. Morgante et al., "Prescribing pattern of anti-Parkinson drugs in southern Italy: cross-sectional analysis in the years 2003-2005," Parkinsonism \& Related Disorders, vol. 14, no. 5, pp. 420-425, 2008.

[7] O. M. Dubaz, S. Wu, F. Cubillos, G. Miao, and T. Simuni, "The Parkinson's foundation quality improvement initiative investigators. Changes in prescribing practices of dopaminergic medications in individuals with Parkinson's disease by expert care centers from 2010 to 2017," Movement Disorders Clinical Practice, vol. 6, no. 8, pp. 687-692, 2019.

[8] W.-M. Liu, R.-M. Wu, C.-H. Chang, J.-W. Lin, Y.-C. Liu, and C.-H. Lin, "National trends of antiparkinsonism treatment in Taiwan: 2004-2011," Parkinson's Disease, vol. 2016, Article ID 1859321, 8 pages, 2016.

[9] PD MED Collaborative Group, "Long-term effectiveness of dopamine agonists and monoamine oxidase $\mathrm{B}$ inhibitors compared with levodopa as initial treatment for Parkinson's disease (PD MED): a large, open-label, pragmatic randomised trial," The Lancet, vol. 384, no. 9949, pp. 1196-1205, 2014.

[10] D. Weintraub, J. Koester, M. N. Potenza et al., "Impulse control disorders in Parkinson disease: a cross-sectional study of 3090 patients," Archives of Neurology, vol. 14, no. 5, 2010.

[11] D. J. Gelb, E. Oliver, and S. Gilman, "Diagnostic criteria for Parkinson disease," Archives of Neurology, vol. 56, no. 1, p. 33, 1999.

[12] A. Antonini and W. Poewe, "Fibrotic heart-valve reactions to dopamine-agonist treatment in Parkinson's disease," The Lancet Neurology, vol. 6, no. 9, pp. 826-829, 2007.

[13] J. A. G. Crispo, A. W. Willis, D. P. Thibault et al., "Associations between anticholinergic burden and adverse health outcomes in Parkinson disease," PLoS One, vol. 11, no. 3, Article ID e0150621, 2016.
[14] K. F. Pedersen, J. P. Larsen, O.-B. Tysnes, and G. Alves, "Natural course of mild cognitive impairment in Parkinson disease," Neurology, vol. 88, no. 8, pp. 767-774, 2017.

[15] S. Mantri, M. Fullard, S. L. Gray et al., "Patterns of dementia treatment and frank prescribing errors in older adults with Parkinson disease," JAMA Neurology, vol. 76, no. 1, p. 41, 2019.

[16] R. E. Heikkila, L. Manzino, F. S. Cabbat, and R. C. Duvoisin, "Protection against the dopaminergic neurotoxicity of 1 methyl-4-phenyl-1,2,5,6-tetrahydropyridine by monoamine oxidase inhibitors," Nature, vol. 311 , no. 5985 , pp. 467-469, 1984.

[17] C. W. Olanow, "Oxidation reactions in Parkinson's disease," Neurology, vol. 40, pp. 37-39, 1990.

[18] C. Mytilineou, P. M. Radcliffe, and C. W. Olanow, "l-(-)-Desmethylselegiline, a metabolite of selegiline [1-(-)-Deprenyl], protects mesencephalic dopamine neurons from excitotoxicity in vitro," Journal of Neurochemistry, vol. 68, no. 1, pp. 434-436, 2002.

[19] C. W. Olanow, R. L. Watts, and W. C. Koller, "An algorithm (decision tree) for the management of Parkinson's disease," Neurology, vol. 56, p. 89, 2001.

[20] Parkinson Study Group, "Effects of tocopherol and deprenyl on the progression of disability in early Parkinson's disease," New England Journal of Medicine, vol. 328, no. 3, pp. 176-183, 1993.

[21] C. W. Olanow, R. A. Hauser, L. Gauguster et al., "The effect of deprenyl and levodopa on the progression of Parkinson's disease," Annals of Neurology, vol. 38, no. 5, pp. 771-777, 1995.

[22] Y. J. Zhao, H. L. Wee, W. L. Au et al., "Selegiline use is associated with a slower progression in early Parkinson's disease as evaluated by Hoehn and Yahr stage transition times," Parkinsonism \& Related Disorders, vol. 17, no. 3, pp. 194-197, 2011.

[23] C. W. Olanow, O. Rascol, R. Hauser et al., "A double-blind, delayed-start trial of rasagiline in Parkinson's disease," New England Journal of Medicine, vol. 361, no. 13, pp. 1268-1278, 2009.

[24] Parkinson Study Group, "Pramipexole vs levodopa as initial treatment for Parkinson disease: a randomized controlled trial," JAMA, vol. 284, no. 15, p. 1931, 2000.

[25] O. Rascol, D. J. Brooks, A. D. Korczyn, P. P. De Deyn, C. E. Clarke, and A. E. Lang, "A five-year study of the incidence of dyskinesia in patients with early Parkinson's disease who were treated with ropinirole or levodopa," New England Journal of Medicine, vol. 342, no. 20, pp. 1484-1491, 2000.

[26] E. Melamed, D. Offen, A. Shirvan, R. Djaldetti, A. Barzilai, and I. Ziv, "Levodopa toxicity and apoptosis," Annals of Neurology, vol. 44, no. 1, pp. S149-S154, 1998.

[27] K. Bhatia, D. J. Brooks, D. J. Burn et al., "Guidelines for the management of Parkinson's disease. The Parkinson's disease consensus working group," Journal of Hospital Medicine, vol. 59, no. 6, pp. 469-480, 1998.

[28] S. Frucht, J. D. Rogers, P. E. Greene, M. F. Gordon, and S. Fahn, "Falling asleep at the wheel: motor vehicle mishaps in persons taking pramipexole and ropinirole," Neurology, vol. 52, no. 9, p. 1908, 1999.

[29] K. R. Chaudhuri, P. Jenner, and A. Antonini, "Should there be less emphasis on levodopa-induced dyskinesia in Parkinson's disease?" Movement Disorders, vol. 34, no. 6, pp. 816-819, 2019.

[30] E. K. Tan, A. P. Yeo, V. Tan, R. Pavanni, and M. C. Wong, "Prescribing pattern in Parkinson's disease: are cost and 
efficacy overriding factors?" International Journal of Clinical Practice, vol. 59, no. 5, pp. 511-514, 2005.

[31] J. M. Pavon, H. E. Whitson, and M. S. Okun, "Parkinson's disease in women: a call for improved clinical studies and for comparative effectiveness research," Maturitas, vol. 65, no. 4, pp. 352-358, 2010.

[32] P. A. Kempster, D. R. Williams, M. Selikhova, J. Holton, T. Revesz, and A. J. Lees, "Patterns of levodopa response in Parkinson's disease: a clinico-pathological study," Brain, vol. 130, no. 8, pp. 2123-2128, 2007. 